\title{
A new paradigm for improved co-ordination and efficacy of European biomedical research: taking diabetes as a model
}

\author{
P. A. Halban • A. J. M. Boulton • U. Smith
}

Received: 12 September 2012 / Accepted: 13 November 2012 /Published online: 13 December 2012

(C) Springer-Verlag Berlin Heidelberg 2012

\begin{abstract}
Today, European biomedical and health-related research is insufficiently well funded and is fragmented, with no common vision, less-than-optimal sharing of resources, and inadequate support and training in clinical research. Improvements to the competitiveness of European biomedical research will depend on the creation of new infrastructures that must be dynamic and free of bureaucracy, involve all stakeholders and facilitate faster delivery of new discoveries from bench to bedside. Taking diabetes research as the model, a new paradigm for European biomedical research is presented, which offers improved co-ordination and common resources that will benefit both academic and industrial clinical research. This includes the creation of a European Council for Health Research, first proposed by the Alliance for Biomedical Research in Europe, which will bring together and consult with all health stakeholders to develop strategic and multidisciplinary research programmes addressing the full innovation cycle. A European Platform for Clinical Research in Diabetes is proposed by the Alliance for European Diabetes Research (EURADIA) in response to the special challenges and opportunities presented by research across the European region, with the need for common standards and shared expertise and data.
\end{abstract}

P. A. Halban ( $\bowtie)$

Department of Genetic Medicine and Development,

University of Geneva Medical Centre, CMU, 1 rue Michel-Servet,

1211 Geneva 4, Switzerland

e-mail: philippe.halban@unige.ch

A. J. M. Boulton

Centre for Endocrinology and Diabetes, University of Manchester,

Manchester, UK

U. Smith

Department of Molecular and Clinical Medicine,

University of Gothenburg, Gothenburg, Sweden
Keywords Clinical research · Diabetes research · Europe · Research infrastructure $\cdot$ Science policy

$\begin{array}{ll}\begin{array}{l}\text { Abbreviations } \\ \text { BioMed Alliance }\end{array} & \begin{array}{l}\text { Alliance for Biomedical Research in } \\ \text { Europe } \\ \text { European Platform for Clinical Research } \\ \text { in Diabetes }\end{array} \\ \text { EU } & \begin{array}{l}\text { European Union } \\ \text { European Council for Health Research } \\ \text { EuCHR }\end{array} \\ \text { EURADIA } & \begin{array}{l}\text { Alliance for European Diabetes } \\ \text { Research } \\ \text { European Commission Seventh }\end{array} \\ & \begin{array}{l}\text { Framework Programme for Research } \\ \text { and Technological Development } \\ (2007-2013) \\ \text { Gross Domestic Product }\end{array} \\ & \end{array}$

Europe is falling behind its traditional competitors in biomedical and health-related research, the USA and Japan, as well as facing increasing competition from newly emerging countries such as China. Lack of co-ordination, inadequate funding (relative to gross domestic product [GDP]) and the complexities inherent in any activity across several countries, each with different commitments and local regulatory constraints, have combined to handicap the European biomedical research enterprise. Although research funding is notoriously difficult to estimate precisely, evaluation by the Organisation for Economic Co-operation and Development (OECD) for the years 20072009 concluded that the average funding for research in the USA was around twice that of the European Union (EU) (\$31 billion vs $\$ 17$ billion per year) [1]. Despite repeated commitments from the European Commission [2], European investment in research is still woefully short of the Commission's own target of 3\% GDP, and much lower than in the USA or Japan [3]. Best estimates for funds devoted exclusively to diabetes 
research in 2008 in the USA and Europe suggest a similar disparity (USA \$1,080 million [4]; Europe $€ 323$ million [5]).

Clinical research is obviously a key component of translational research, embracing all studies on living individuals as well as safety and efficacy studies on new therapeutic molecules. Despite the impressive history of clinical research in Europe, with major contributions to our understanding of diseases and the development of innovative therapeutic approaches for their treatment, the situation today is worrisome. As always in times of financial constraint, health and education, including research, will be among the first to suffer from budget cuts. Indeed, at the time of writing there is political uncertainty surrounding the budget for Horizon 2020 (the EU Framework Programme for Research and Innovation for the period 2014-2020) with the serious risk of a substantial decrease in overall funding. National funding of research will certainly also suffer in many European countries. We must therefore find new ways to make biomedical research in general, and clinical research in particular, not only more cost-effective but also more competitive, offering more to society in return for investment, while allowing equal access to high-quality clinical studies for all citizens in Europe. Clinical research is undertaken both by academia and the pharmaceutical industry, albeit generally for different reasons. Faced with increasingly challenging demands from regulatory agencies and the spiralling costs of clinical studies, pharmaceutical companies would also benefit greatly from any common infrastructure that offered greater efficacy and improved co-ordination of clinical research.

\section{European Council for Health Research}

Strategic planning across disciplines and involving all major health stakeholders is required to enable the realisation of the EU's 'Innovation Union' initiative [6]. This aims to create an environment in Europe that supports the translation of novel ideas into products and services, can generate growth and jobs, and improve well-being. Biomedical research represents an outstanding example of research-driven innovation that can promote future growth.

For the first time, the European biomedical community has come together as an 'Alliance for Biomedical Research in Europe', which already represents 21 major health research organisations and around 250,000 scientists (www.biomedeurope.org/). The BioMed Alliance is calling on EU and national decision-makers in Europe for urgent action to help science achieve true innovation that can create a healthier and more prosperous environment for Europe's citizens. The BioMed Alliance is forcefully promoting the creation of the European Council for Health Research (EuCHR), which can provide the best scientific leadership to EU programmes in health research and will bring together and consult with all health stakeholders. The overall goal is to accelerate research in the biomedical field in Europe through long-term strategic research programmes capable of triggering multidisciplinary partnerships and addressing the full innovation cycle. As shown in Fig. 1, translational research ('from bench to bedside') involves three major levels of research (Fig. 1, black), with multiple performance sites (Fig. 1, red) and both public and private sources of funding (Fig. 1, blue). The EuCHR would allow for coordination of this complex enterprise that is presently missing in Europe, thereby speeding delivery of new drugs to patients while making this more cost-effective. At the time of writing the EuCHR is being discussed with the European Commission Directorate General for Research and Innovation and key members of the European Parliament. It is hoped that this council can be established to coincide with the Horizon 2020 programme.

\footnotetext{
Aims of the European Council for Health Research (EuCHR)

- Accelerate excellent biomedical and health-related research in Europe

- Seek better co-ordination and strategic planning of biomedical research funding programmes

- Define and support high-level research programmes in order to achieve translation and innovative outcomes

- Promote deeper and longer-term collaborative initiatives that address current gaps in the innovation cycle

- Provide strategic advice on the steering of European health research to policy makers

- Incorporate all relevant stakeholders in order to boost creativity and innovation
} 


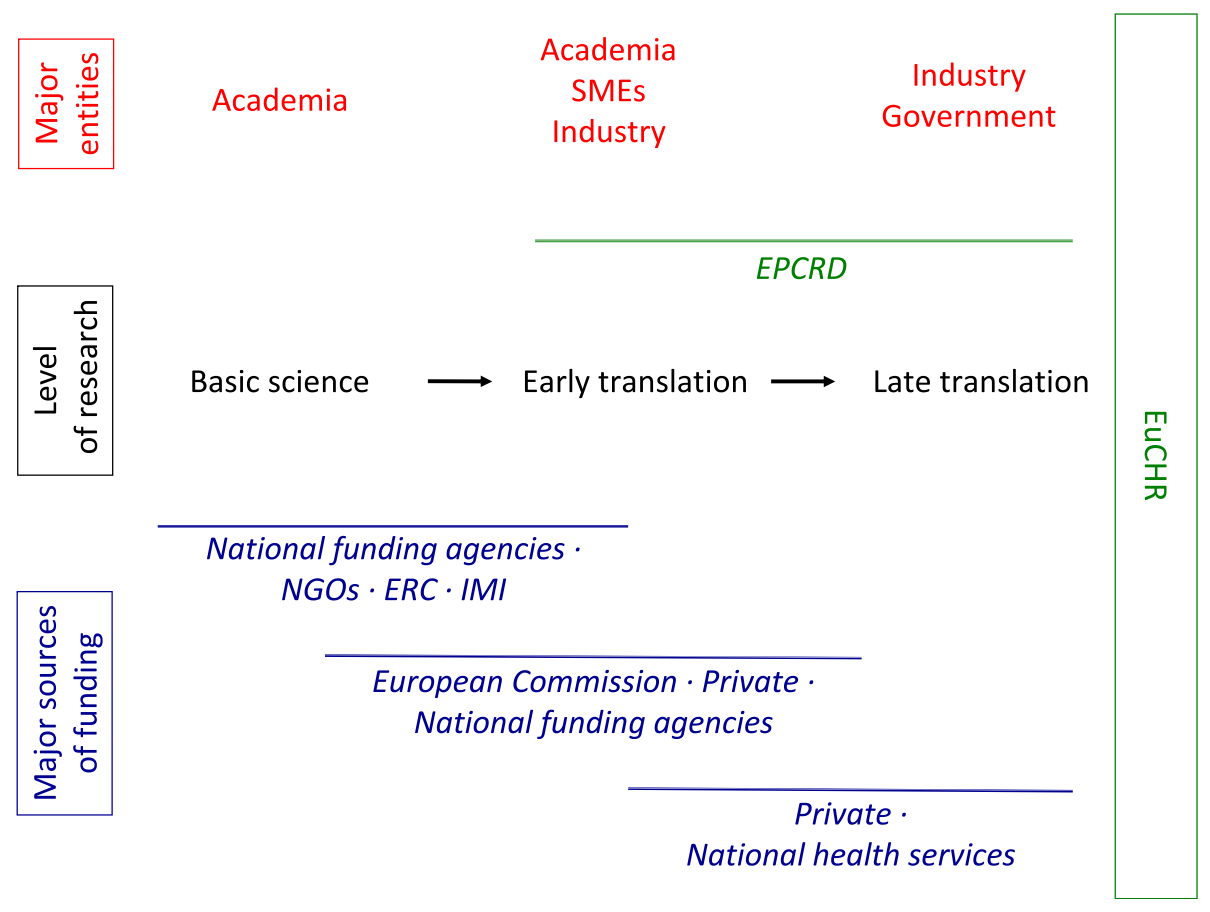

Fig. 1 European translational research landscape. This scheme indicates the current complexity and fragmentation of European biomedical research, with multiple public and private stakeholders operating independently. Major entities performing research are shown in red, the level (or stage) of research in black, and funding sources in blue. European Commission funding would include the current FP7 and the future Horizon 2020 programme supported by the Directorate General for Research and Innovation. Private support is from industry or SMEs (small and medium enterprises). Specific examples of funding

\section{European Platform for Clinical Research in Diabetes}

The DIAMAP project, a roadmap for European diabetes research, was supported by a grant from the European Commission Seventh Framework Programme (FP7) and submitted its report in 2010 (full report and a summary brochure at www.diamap.eu [7]). A major recommendation was to create a European Platform for Clinical Research in Diabetes (EPCRD). Although focused on a single disease, the fact that diabetes, together with its macrovascular and microvascular complications and comorbidities, is of common interest to most members of the BioMed Alliance makes the concept and rationale for such a platform directly in line with the major goals of the EuCHR; indeed, the EPCRD was used as one of only a handful of examples in support of the BioMed Alliance's White Paper on the creation of the EuCHR. The Alliance for European Diabetes Research (EURADIA; www.euradia.org), the coordinator of sources are provided for illustrative purposes only and should not be considered exhaustive. Coordinating structures described further in the text are shown in green. The EuCHR proposed by the BioMed Alliance is intended to coordinate all biomedical research activities in Europe. Clinical research platforms, such as the EPCRD under development by EURADIA, would offer the public and private sectors improved efficacy and competitiveness. ERC, European Research Council; IMI, Innovative Medicines Initiative; NGO, non-governmental organisation

DIAMAP, intends to promote the development of the EPCRD.

Rationale The DIAMAP road maps repeatedly mentioned as roadblocks the need for registries of people with diabetes, networks of specialist researchers, access to biobanks and human biological material (especially in relation to the rarer complications) and more standardised evidence-based treatment guidelines. Lack of political and public understanding of biomedical research and its importance to society was also cited as a roadblock, with the recommendation for better education leading to greater involvement of people with diabetes in clinical research; their support is essential and offers a significant opportunity for transparency in determining how diabetes research budgets are spent. The EPCRD will for the first time in Europe offer equal access to clinical research to all people with diabetes, across all traditional borders of language, culture and education. It was 


\section{European Platform for Clinical Research in Diabetes (EPCRD)}

\section{Aims}

- Facilitate and enhance clinical diabetes research with the purpose of improving care and treatment for people with diabetes

\section{Objectives}

- Create centrally determined governance structures in line with current ethical guidelines

- Facilitate access to information and online databases of clinical studies and trials, thus encouraging participation by interested volunteers (with diabetes and without). The closer dialogue between professionals and research participants is intended to encourage greater understanding of the science

- Improve access to structured education and training for European diabetes researchers and healthcare professionals engaging in research activity

- Improve access to information and structured education for people living with diabetes

- Facilitate access to data and biological samples by providing a uniformly agreed and ethically approved infrastructure to permit sample and data sharing across multiple national and international security barriers

- Encourage investment by and participation of industry, facilitating access to a large number of research subjects and to scientists from subspecialties. Funding of industry-initiated trials could be standardised across Europe supporting the concept of the 'European diabetes patient'. The use of such a market approach to clinical research has the potential to drive down costs to increase the competitiveness of Europe as a clinical trial location

further felt that diabetes research would be enhanced if the clinical research community itself could drive a collaborative initiative as it deals with the consequences of research on treatment and care delivery. EURADIA is hoping to create the platform in 2013 and will support a research project to test the system before it is made available to all stakeholders as a fully integrated component of a new landscape for European clinical research (Fig. 1).

\section{Conclusions}

There is an urgent need for an umbrella organisation to oversee and coordinate biomedical and health-related research in Europe. This organisation would ideally be independent of European and national funding agencies but supported by them and fully integrated into a truly regional research operation. We believe that the EuCHR, as proposed by the BioMed Alliance, would satisfy all these requirements, offering in return to the European Commission, national governments and most importantly the citizens of Europe, a healthier future thanks to more effective translational research and improved return on investment for society at large, with more jobs and greater innovation. The proposed EPCRD would be just one activity of the EuCHR among many others, serving as a model for all disease areas.

This new paradigm for biomedical research in Europe, taking diabetes as the model case, will only become a reality if it receives proactive support from all stakeholders. Researchers, health professionals, private enterprises, non-governmental organisations and, last but by no means least, people with diabetes and their families, should advocate at the local, national and regional levels to ensure that there will be political and budgetary support for the creation of the proposed infrastructure. The EASD, a founding member of both the BioMed Alliance and of EURADIA, and its foundation (the EFSD), the leading pan-European diabetes research funding agency, are uniquely well placed to promote this bold new action plan.

Acknowledgements Andrew Boulton is President of the EASD and the EFSD; Philippe Halban is Chairman of EURADIA and Honorary Chairman of the EFSD; Ulf Smith is President of the BioMed Alliance and Chairman of the EFSD. Any opinions expressed here are those of the authors and do not necessarily reflect those of their respective organisations.

Duality of interest The authors declare that there is no duality of interest associated with this manuscript.

Contribution statement All authors contributed to the conception, drafting and final approval of this article. 


\section{References}

1. Berghmans S, Bisangi A, Bouillon R et al (2011) European Medical Research Councils: White Paper II: A stronger biomedical research for a better European future. Available from www.esf.org/ fileadmin/FlipBooks/index_emrc_white_paperII.html\#/1/, accessed 12 September 2012

2. European Commission European Research Area: Investing in European Research: the 3\% objective. Available from http:// ec.europa.eu/research/era/areas/investing/investing_research_ en.htm, accessed 15 November 2012

3. European Commission (2012) Eurostat R \& D expenditure. Available from http://epp.eurostat.ec.europa.eu/statistics_explained/
index.php/R_\%26_D_expenditure\#Further_Eurostat_information, accessed 12 September 2012

4. National Institutes of Health (2012) NIH Budget. Available from www.nih.gov/about/budget.htm, accessed 12 September 2012

5. DIAMAP (2010) Road map report: strategic plan for diabetes research: Chapter 9, Funding survey. Available from DIAMAP funding survey. www.diamap.eu/uploads/pdf/DIAMAP-Chapter-9.pdf, accessed 12 September 2012

6. European Commission Innovation Union (2012). Available from http://ec.europa.eu/research/innovation-union/index en.cfm? pg=intro, accessed 15 November 2012

7. DIAMAP (2010) Road map report: strategic plan for diabetes research. Available from http://www.diamap.eu, accessed 12 September 2012 